\title{
SEMI-INFINITE PATHS OF THE TWO-DIMENSIONAL RADIAL SPANNING TREE
}

\author{
FRANÇOIS BACCELLI, ${ }^{*}$ INRIA \\ DAVID COUPIER $* * * * *$ AND \\ VIET CHI TRAN, ${ }^{* *}$ Université Lille1
}

\begin{abstract}
We study semi-infinite paths of the radial spanning tree (RST) of a Poisson point process in the plane. We first show that the expectation of the number of intersection points between semi-infinite paths and the sphere with radius $r$ grows sublinearly with $r$. Then we prove that in each (deterministic) direction there exists, with probability 1 , a unique semi-infinite path, framed by an infinite number of other semi-infinite paths of close asymptotic directions. The set of (random) directions in which there is more than one semi-infinite path is dense in $[0,2 \pi)$. It corresponds to possible asymptotic directions of competition interfaces. We show that the RST can be decomposed into at most five infinite subtrees directly connected to the root. The interfaces separating these subtrees are studied and simulations are provided.
\end{abstract}

Keywords: Stochastic geometry; random tree; semi-infinite path; asymptotic direction; competition interface

2010 Mathematics Subject Classification: Primary 60D05

\section{Introduction}

In this paper, we are interested in semi-infinite paths of the two-dimensional radial spanning tree (RST) introduced in [2]. Let us consider a homogeneous Poisson point process (PPP) $N$ on $\mathbb{R}^{2}$ (endowed with its usual Euclidean norm $|\cdot|$ ) with intensity 1 . Throughout this paper, $N$ is considered in its Palm version: it almost surely (a.s.) contains the origin $O$. The RST is a random graph $\mathcal{T}(N)$ (or merely $\mathcal{T}$ ) defined as follows. Its vertex set is $N$. Its edge set $E$ contains each pair $\{X, Y\}, X, Y \in N$ and $X \neq O$, such that

$$
|Y|<|X| \quad \text { and } \quad N \cap B(O,|X|) \cap B(X,|X-Y|)=\varnothing
$$

(where $B(c, r)$ denotes the open ball with center $c$ and radius $r$ ). For any $X \in N \backslash\{O\}$, there is a.s. only one $Y \in N$ satisfying (1.1). Among the vertices of $N \cap B(O,|X|)$, this is the closest to $X$. This vertex is denoted by $\mathcal{A}(X)$ and called the ancestor of $X$. With an abuse of notation, for an edge $e$, we call the ancestor of the edge the endpoint that is the ancestor of the other endpoint, which we call the descendant of the edge. The ancestor of $e$ is the endpoint of $e$ that is closer from $O$. With probability 1 , the graph $\mathcal{T}$ admits a tree structure (there is no loop) rooted at the origin $O$. For convenience, we set $\mathcal{A}(O)=O$.

Received 12 June 2012; revision received 12 October 2012.

* Postal address: Research group on Network Theory and Communications (TREC), INRIA-ENS, 75214 Paris, France.

** Postal address: Laboratoire Paul Painlevé, Université Lille 1, Cité Scientifique, 59655 Villeneuve d'Ascq Cedex, France.

*** Email address: david.coupier@math.univ-lille1.fr 
A sequence $\left(X_{n}\right)_{n \geq 0}$ of vertices of $N$ is a semi-infinite path of the RST $\mathcal{T}$ if, for any $n, X_{n}$ is the ancestor of $X_{n+1}$. A semi-infinite path $\left(X_{n}\right)_{n \geq 0}$ has asymptotic direction $\theta \in[0,2 \pi)$ if

$$
\lim _{n \rightarrow \infty} \frac{X_{n}}{\left|X_{n}\right|}=\mathrm{e}^{\mathrm{i} \theta}
$$

(by identifying $\mathbb{R}^{2}$ with the complex plane $\mathbb{C}$ ).

Radial random trees in the plane have been studied in many other papers, including [3], [13], and [14]. The main difficulty in the case of the RST is that the local rule (1.1) used for selecting the ancestor implies complex dependencies. The latter make it difficult to exhibit natural Markov processes and prevent a direct use of martingale convergence theorems or Lyapunov functions. However, our study can rely on the following result of [2, Theorem 2.1] on the RST.

Theorem 1.1. The following properties hold a.s.

(i) Every semi-infinite path of $\mathcal{T}$ has an asymptotic direction.

(ii) For every $\theta \in[0,2 \pi)$, there exists at least one semi-infinite path with asymptotic direction $\theta$.

(iii) The set of $\theta \sin [0,2 \pi)$ such that there is more than one semi-infinite path with asymptotic direction $\theta$ is dense in $[0,2 \pi)$.

This result is based on a clever method due to Howard and Newman [11, Section 2.3] proving that the above properties hold for any deterministic tree that satisfies some straightness condition, which is shown to be a.s. satisfied for the RST (see [2, Theorem 5.4]).

Understanding the finer structural properties of radial random trees, such as the asymptotic directions of their infinite branches or the shape of the interfaces that separate subtrees, has been a recurrent research topic. See, for instance, [4], [6], and [5] for results on the geodesics and interfaces of the last passage percolation tree. The aim of the present paper is to investigate such properties for the RST.

Our first result concerns the number of intersection points between semi-infinite paths of the RST $\mathcal{T}$ and the sphere with radius $r$ centered at the origin; its expectation tends to $\infty$ but slower than $r$ (Theorem 2.1). The proof is based on the local approximation of the RST, far enough from the origin, by the directed spanning forest (DSF). See [2] for details. Moreover, it has been proved recently (see [7, Theorem 8]) that there is no bi-infinite path in the DSF. This allows us to conclude.

We then focus our attention on semi-infinite paths with deterministic directions. Proposition 3.1 states that, for any given $\theta \in[0,2 \pi)$, there is a.s. exactly one semi-infinite path with direction $\theta$. Let $\gamma_{0}$ be the path corresponding to $\theta=0$. Proposition 3.1 provides a further description of the subtree of the RST made up of $\gamma_{0}$ and all the branches emanating from it.

Finally, we study the subtrees of the RST rooted at the children of $O$. To do so, each of these subtrees is painted a different color. This process produces the colored RST. Each of these colored subtrees can be bounded or not. Since the origin can have at most five descendants with probability 1 , there are at most five distinct unbounded subtrees rooted at $O$. We prove in Theorem 4.1 that their number may be equal to $1,2,3,4$, or 5 with positive probability. The border between two colored subtrees is called the competition interface. Any unbounded competition interface admits an asymptotic direction (Proposition 4.1). This direction is random and corresponds to one of (at least) two semi-infinite paths, as in part (iii) of Theorem 1.1. 
It is worth pointing out here that our proofs strongly rely on the planarity of the RST and the noncrossing property of its branches (see Lemma A.1 in Appendix A). They cannot be extended to arbitrary dimensions.

The paper is organized as follows. In Section 2, the sublinear character of the expected number of intersection points between semi-infinite paths and the sphere with radius $r$ is established. Section 3 contains results on semi-infinite paths with deterministic directions. The colored RST and competition interfaces are defined in Section 4. Finally, open questions and numerical studies are gathered in Section 5.

\section{Sublinearity of the number of semi-infinite paths}

Let $r$ be a positive real number. Let us denote by $\chi_{r}$ the number of intersection points of the sphere $S(O, r)=\left\{r \mathrm{e}^{\mathrm{i} \theta}, \theta \in[0,2 \pi)\right\}$ with the semi-infinite paths of the RST. The main result of this section states that the expectation of $\chi_{r}$ is sublinear.

Theorem 2.1. The following limit holds:

$$
\lim _{r \rightarrow \infty} \mathbb{E}\left(\frac{\chi_{r}}{r}\right)=0
$$

The idea of the proof is as follows. For $r>0$, we introduce the points $A_{r}=r \mathrm{e}^{\mathrm{i} / r}$ and $B_{r}=r \mathrm{e}^{-\mathrm{i} / r}$ of the sphere $S(O, r)$. In the sequel we will denote by $\left[A_{r}, B_{r}\right]$ the line segment with extremities $A_{r}$ and $B_{r}$, and by $a\left(A_{r}, B_{r}\right)=\left\{r \mathrm{e}^{\mathrm{i} \theta}, \theta \in[-1 / r, 1 / r]\right\}$ the $\operatorname{arc}$ of $S(O, r)$ with extremities $A_{r}$ and $B_{r}$, containing the point $(r, 0)$. This arc is, by construction, of length 2 . We denote by $\tilde{\chi}_{r}$ the number of intersection points between semi-infinite paths of the RST and $a\left(A_{r}, B_{r}\right)$. By the rotational invariance of the PPP $N, \mathbb{E}\left(\chi_{r}\right)=\pi r \mathbb{E}\left(\tilde{\chi}_{r}\right)$. Hence, using an additional moment condition, the proof of Theorem 2.1 will be shown to amount to proving that

$$
\lim _{r \rightarrow \infty} \mathbb{P}\left(\tilde{\chi}_{r} \geq 1\right)=0 .
$$

To prove (2.1), note that, far enough from the origin, the RST can be locally approximated by the DSF (see [2]). The DSF $\mathcal{T}_{-e_{x}}$ with direction $-e_{x}=-(1,0)$ is a graph built on the PPP $N$ and in which each vertex $X$ has as ancestor the closest point of $N$ among those with strictly smaller abscissa. This construction generates a family of trees, i.e. a forest, which bears similarities with other directed forests introduced in the literature (see, e.g. [1], [8], and [9]). As $r$ tends to $\infty$, the neighborhood of $(r, 0)$ in the RST increasingly looks similar to the neighborhood of $O$ in the DSF. Hence, the probability $\mathbb{P}\left(\tilde{\chi}_{r} \geq 1\right)$ that there exists an infinite path crossing $a\left(A_{r}, B_{r}\right)$ is close to that of having a path of the DSF crossing $\{0\} \times[-1,1]$ that is very long in the direction $e_{x}$. Such a phenomenon is rare since the DSF is known to a.s. have only one topological end [7].

In order to prove Theorem 2.1, we will need the two following lemmas whose proofs are deferred to the end of the section.

Lemma 2.1. For any $r>0$, the number of edges of the RST that intersect an arc of $S(O, r)$ of length 1 has finite second-order moment and, moreover,

$$
\limsup _{r \rightarrow+\infty} \mathbb{E}\left(\tilde{\chi}_{r}^{2}\right)<+\infty .
$$

Lemma 2.2 below specifies how the RST is approximated by the DSF: far from the origin (around the point $(r, 0))$ and locally (for the neighborhood of radius $r^{\alpha}$ of $(r, 0)$ ). Note that, 
since the distribution of the DSF is invariant by translation along $e_{x}, \mathcal{T}_{-e_{x}} \cap B\left((r, 0), r^{\alpha}\right)$ and $\mathcal{T}_{-e_{x}} \cap B\left(O, r^{\alpha}\right)$ have the same distribution.

Lemma 2.2. Let $\mathcal{T}$ and $\mathcal{T}_{-e_{x}}$ respectively denote the $R S T$ and DSF of direction $-e_{x}$ constructed on the same PPP N. Then, for $0<\alpha<\frac{1}{3}$,

$$
\lim _{r \rightarrow+\infty} \mathbb{P}\left(\mathcal{T} \cap B\left((r, 0), r^{\alpha}\right)=\mathcal{T}_{-e_{x}} \cap B\left((r, 0), r^{\alpha}\right)\right)=1 .
$$

The approximation also holds if we replace $r^{\alpha}$ by a constant radius $R$.

Proof of Theorem 2.1. Step 1. Let us prove (2.1). First, note that all the paths which intersect the arc $a\left(A_{r}, B_{r}\right)$ necessarily intersect the segment $\left[A_{r}, B_{r}\right]$ (the converse is not necessarily true). The segment $\left[A_{r}, B_{r}\right]$ is perpendicular to the horizontal axis, and all its points have abscissa $\tilde{r}=r \cos (1 / r)$. Its length is $2 r \sin (1 / r) \leq 2$.

Heuristically, the event $\left\{\tilde{\chi}_{r} \geq 1\right\}$ (consisting of the existence of at least one semi-infinite path crossing $\left.a\left(A_{r}, B_{r}\right)\right)$ is hence close to the existence of a path of the RST crossing the vertical segment $\left[A_{r}, B_{r}\right]$ and then surviving until it has a large radius. For $R>0$, let us therefore consider the event where there exists a path of the RST crossing $\{\tilde{r}\} \times[-1,1] \supset\left[A_{r}, B_{r}\right]$ before intersecting the sphere $S((\tilde{r}, 0), R)$. Our aim is to show that the probability of this event is close to the probability that in the DSF $\mathcal{T}_{-e_{x}}$ there exists a path intersecting $\{0\} \times[-1,1]$ and then $S(O, R) \cap\{x>0\}$. To show that such an approximation holds, let us prove that our event is local in the sense of Lemma 2.2 .

Let us consider a path of the RST first crossing $\{\tilde{r}\} \times[-1,1]$ and then $S((\tilde{r}, 0), R)$, i.e. towards descendants, as described below. From this path, we can extract a subpath $\left(Z_{0}, \ldots, Z_{n}\right)$ crossing only once $\{\tilde{r}\} \times[-1,1]$ (between $Z_{0}$ and $\left.Z_{1}\right)$ and $S((\tilde{r}, 0), R)$ (between $Z_{n-1}$ and $Z_{n}$ ). See Figure 1. We show that this path is included in the ball $B((\tilde{r}, 0), 2 R)$, so that the local approximation of the RST by the DSF (see Lemma 2.2) holds. If $Z_{0}$ is outside the ball $B((\tilde{r}, 0), R)$ then $B\left(Z_{1},\left|Z_{1}-Z_{0}\right|\right)$ contains $B((\tilde{r}, 0), R / 2)$ for large enough $R$. Consequently, the set $B(O, \tilde{r}) \cap B((\tilde{r}, 0), R / 2)$ is empty of points of the PPP $N$. So, $Z_{0}$ belongs to $B((\tilde{r}, 0), R)$ with high probability as $r$ and $R$ tend to $\infty$ (with $r$ tending to $\infty$ faster than $R$ ). The same is true about the endpoint $Z_{n}$ and the ball $B((\tilde{r}, 0), 2 R)$. To sum up, given $\varepsilon>0$ and large

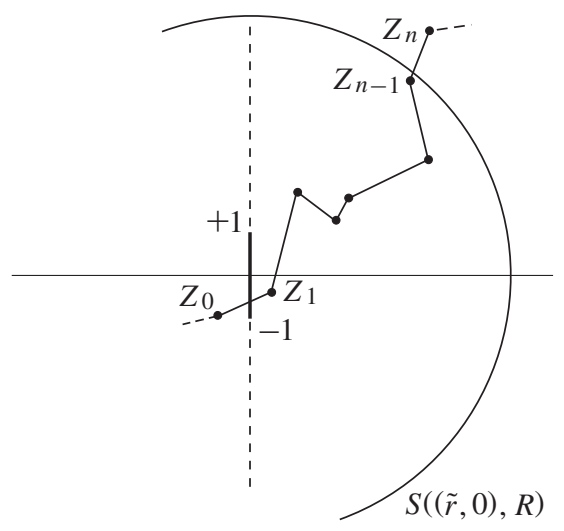

FIgURE 1: The subpath $\left(Z_{0}, \ldots, Z_{n}\right)$ of the RST crossing the vertical segment $\{\tilde{r}\} \times[-1,1]$ (thick line) and the sphere $S((\tilde{r}, 0), R)$. Here $Z_{0}$ and $Z_{n}$ belong to $B((\tilde{r}, 0), 2 R)$, which occurs with high probability. 
enough $r, R$,

$$
\begin{aligned}
& \mathbb{P}\left(\tilde{\chi}_{r} \geq 1\right) \leq \mathbb{P}(\text { there exists a path of the RST that first crosses }\{\tilde{r}\} \times[-1 ; 1] \text { and } \\
&\text { then } S((\tilde{r}, 0), R), \text { whose endpoints belong to } B((\tilde{r}, 0), 2 R))+\varepsilon \\
& \leq \mathbb{P}(\text { there exists a path of the DSF that first crosses }\{0\} \times[-1 ; 1] \text { and } \\
&\text { then } S(O, R))+2 \varepsilon .
\end{aligned}
$$

Theorem 8 of [7] states that each path of the DSF is a.s. finite towards descendants. Then the probability on the right-hand side of (2.2) tends to 0 as $R$ tends to $\infty$. This means that $\mathbb{P}\left(\tilde{\chi}_{r} \geq 1\right)$ is smaller than $3 \varepsilon$, i.e. $(2.1)$.

Step 2. Let us prove that $\mathbb{E}\left(\chi_{r}\right)$ is sublinear. As mentioned at the beginning of the section, it is sufficient to show that $\lim _{r \rightarrow \infty} \mathbb{E}\left(\tilde{\chi}_{r}\right)=0$.

Since, by the Cauchy-Schwarz inequality,

$$
\mathbb{E}\left(\tilde{\chi}_{r}\right) \leq \sqrt{\mathbb{E}\left(\tilde{\chi}_{r}^{2}\right)} \sqrt{\mathbb{P}\left(\tilde{\chi}_{r} \geq 1\right)},
$$

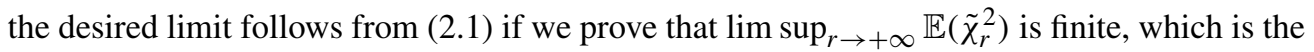
result of Lemma 2.1.

We end this section with the proofs of Lemmas 2.1 and 2.2.

Proof of Lemma 2.1. Let $A_{r}$ and $B_{r}$ be as in the proof of Theorem 2.1. Let $W_{r}$ be the intersection point of the two tangents to $S(O, r)$ that pass through the points $A_{r}$ and $B_{r}$. See the left-hand diagram of Figure 2.

The number of semi-infinite paths that cross $a\left(A_{r}, B_{r}\right)$ is upper bounded by the number $\check{\chi}_{r}+\widehat{\chi}_{r}$ of edges of the RST which intersect $\left[A_{r}, W_{r}\right] \cup\left[W_{r}, B_{r}\right]$, where $\check{\chi}_{r}$ and $\widehat{\chi}_{r}$ respectively denote the numbers of edges crossing $\left[A_{r}, W_{r}\right]$ and $\left[W_{r}, B_{r}\right]$, whose ancestors belong to the same half-plane delimited by the line supporting $\left[A_{r}, W_{r}\right]$ and $\left[W_{r}, B_{r}\right]$, respectively, as $O$. Since $\check{\chi}_{r}$ and $\widehat{\chi}_{r}$ are identically distributed,

$$
\mathbb{E}\left(\tilde{\chi}_{r}^{2}\right) \leq \mathbb{E}\left(\left(\check{\chi}_{r}+\widehat{\chi}_{r}\right)^{2}\right) \leq 4 \mathbb{E}\left(\check{\chi}_{r}^{2}\right),
$$

and it is sufficient to show that $\lim _{\sup } \rightarrow \infty \mathbb{E}\left(\check{\chi}_{r}^{2}\right)$ is finite. By rotational invariance, the distribution of $\check{\chi}_{r}$ is also the distribution of the number of edges with ancestors of smaller
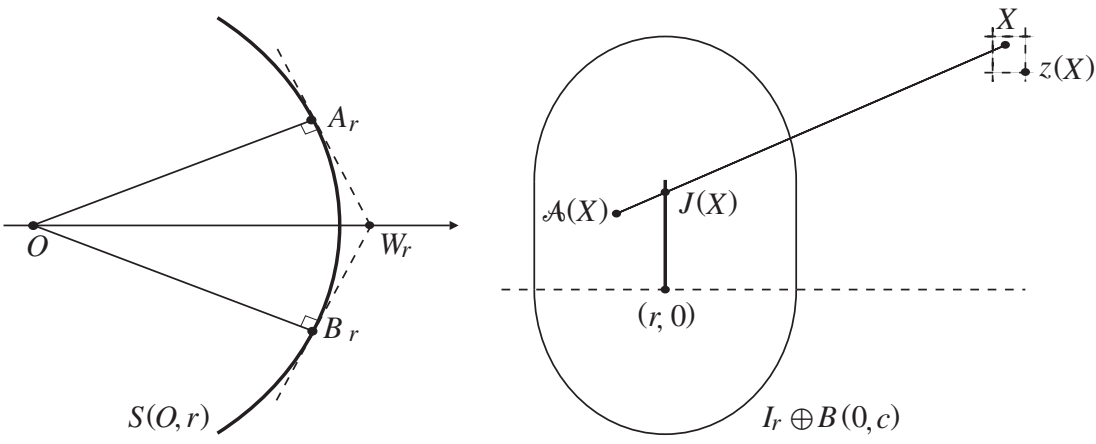

Figure 2: Left: the sphere $S(O, r)$ with center $O$ and radius $r$ is depicted by a thick line. The tangents (dashed lines) to $S(O, r)$ at $A_{r}$ and $B_{r}$ intersect the horizontal axis at $W_{r}$. Right: the segment $[X, \mathcal{A}(X)]$ crosses $I_{r}$ (thick line) on $J(X)$. Here $X$ is outside $I_{r} \oplus B(0, c)$. 
abscissa and that cross the vertical segment $I_{r}=[(r, r \tan (1 / r)),(r, 0)]$. With an abuse of notation, we will again denote by $\check{\chi}_{r}$ the last random variable. Also, note that the length of $I_{r}$ is bounded by 2 as soon as $r$ is sufficiently large. Let $c>2+3 \sqrt{2}$ (a technical condition needed in the sequel), and let us use ' $\oplus$ ' for the Minkowski addition. Then

$$
\check{\chi}_{r}=\check{\chi}_{r}^{\leq c}+\check{\chi}_{r}^{>c} \quad \text { a.s. }
$$

where $\check{\chi}_{r}^{\leq c}$ (respectively $\check{\chi}_{r}^{>c}$ ) denotes the number of these edges with descendants belonging to $I_{r} \oplus B(O, c)$ (respectively being a distance at least $c$ from $I_{r}$ ). The number $\check{\chi}_{r}^{\leq c}$ is upper bounded by $\operatorname{card}\left(N \cap\left(I_{r} \oplus B(O, c)\right)\right)$ and admits a moment of order 2 that is bounded independently of $r$. It remains to study $\check{\chi}_{r}^{>c}$. Our idea is that each long edge is accompanied by a large empty space, so that it is rare that many long edges intersect $I_{r}$.

Let us consider an edge $[\mathcal{A}(X), X]$ that crosses $I_{r}$ at $J(X)$ and such that the distance from $X$ to $I_{r}$ is larger than $c$. If $X=(x, y)$ then let us consider $z(X)$ the point with coordinates $([x]+1, \operatorname{sgn}(y)[|y|])$, where $[x]$ denotes the integer part of $x$. Among the points with integer coordinates that have an abscissa larger than $x$ and which are closer to the abscissa axis than $X, z(X)$ is the point that is the closest to $X$. See the right-hand diagram of Figure 2 .

By construction, $|X-z(X)| \leq \sqrt{2}$. Hence, $B(O,|z(X)|-\sqrt{2}) \subset B(O,|X|)$, where the radius of the first ball is positive as soon as $r \geq \sqrt{2}$. Let us consider the ball $B(z(X)$, $|z(X)-(r, 0)|-2-2 \sqrt{2})$. For our choice of $c$,

$$
|z(X)-(r, 0)|-2-2 \sqrt{2} \geq c-\sqrt{2}-2-2 \sqrt{2} \geq 0
$$

and the radius is positive. If $U \in B(z(X),|z(X)-(r, 0)|-2-2 \sqrt{2})$ then

$$
|U-X| \leq|U-z(X)|+\sqrt{2} \leq|z(X)-(r, 0)|-2-\sqrt{2} \leq|X-J(X)| \leq|X-\mathcal{A}(X)|
$$

and, thus, $B(z(X),|z(X)-(r, 0)|-2-2 \sqrt{2}) \subset B(X,|X-\mathcal{A}(X)|)$. As a consequence, if we introduce

$$
\Lambda(z, r)=B(O,|z|) \cap B(z,|z-(r, 0)|-2-2 \sqrt{2})
$$

for $z=\left(z_{x}, z_{y}\right) \in \mathbb{Z}^{2}$ and sufficiently large $r$, we have

$$
\operatorname{card}(N \cap \Lambda(z(X), r)) \leq \operatorname{card}(N \cap B(O,|X|) \cap B(X,|X-\mathcal{A}(X)|)),
$$

the latter quantity being 0 since $[\mathcal{A}(X), X]$ is an edge of the RST, implying that there is no point of $N$ in $\Lambda(z(X), r)$. Thus, for $r \geq \sqrt{2}$,

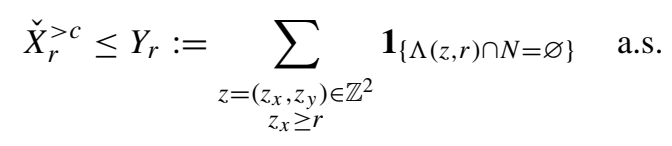

Note that if $r$ and $r^{\prime}$ are such that $z_{x} \geq r^{\prime} \geq r$, then $\Lambda(z, r) \subset \Lambda\left(z, r^{\prime}\right)$. This implies that $r \mapsto Y_{r}$ is a.s. a decreasing function of $r$. Then, if we fix $r_{0} \geq \sqrt{2}$ for all $r \geq r_{0}, \check{X}_{r}^{>c} \leq Y_{r_{0}}$. The volume of $\Lambda\left(z, r_{0}\right)$ is of the order of $|z|^{2}$ and, for a given integer $\rho \geq r_{0}^{2}$, the number of points $z$ such that $|z|^{2}=\rho$ is of the order of $\sqrt{\rho}$. Thus, for two positive constants $C$ and $C^{\prime}$,

$$
\mathbb{E}\left(Y_{r_{0}}\right)=\sum_{\substack{z=\left(z_{x}, z_{y}\right) \in \mathbb{Z}^{2} \\ z_{x} \geq r_{0}}} \mathbb{P}\left(\Lambda\left(z, r_{0}\right) \cap N=\varnothing\right) \leq C \sum_{\rho \geq r_{0}^{2}} \sqrt{\rho} \mathrm{e}^{-C^{\prime} \rho}<+\infty .
$$


It now remains to prove that $\mathbb{E}\left(Y_{r_{0}}^{2}\right)<+\infty$. For this, we compute

$$
\begin{aligned}
& \mathbb{E}\left(Y_{r_{0}}\left(Y_{r_{0}}-1\right)\right)=\sum_{\substack{z=\left(z_{x}, z_{y}\right) \in \mathbb{Z}^{2} \\
z^{\prime}=\left(z_{x}^{\prime}, z_{y}^{\prime}\right) \in \mathbb{Z}^{2} \\
z_{x} \geq r_{0}, z_{x}^{\prime} \geq r_{0} \\
z_{x} \neq z_{x}^{\prime}}} \mathbb{E}\left(\mathbf{1}_{\left\{\Lambda\left(z, r_{0}\right) \cap N=\varnothing\right\}} \mathbf{1}_{\left.\left\{\Lambda\left(z^{\prime}, r_{0}\right) \cap N=\varnothing\right\}\right)}\right.
\end{aligned}
$$

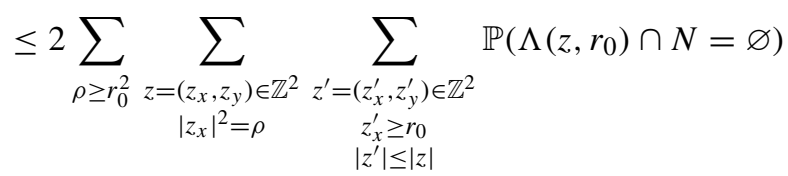

$$
\begin{aligned}
& \leq C \sum_{\rho \geq r_{0}^{2}} \rho^{3 / 2} \mathrm{e}^{-C^{\prime} \rho} \\
& <+\infty
\end{aligned}
$$

for two positive constants $C$ and $C^{\prime}$. Equations (2.3) and (2.4) show that $\mathbb{E}\left(Y_{r_{0}}^{2}\right)<+\infty$, and this concludes the proof.

Proof of Lemma 2.2. We follow here the proof of Baccelli and Bordenave [2, Section 3.6], where the case of a fixed radius $R$ is considered. Recall that $\mathcal{T}$ and $\mathcal{T}_{-e_{x}}$ are the RST and DSF with direction $-e_{x}$, constructed on the same PPP $N$. We denote by $\mathcal{A}(X)$ and $\mathcal{A}_{-} e_{x}(X)$ the ancestors of $X$ in $\mathcal{T}$ and $\mathcal{T}_{-e_{x}}$. Let $r>0, \alpha>0$, and $\beta>0$. Then

$$
\begin{aligned}
\mathbb{P}(\mathcal{T} & \left.\cap B\left((r, 0), r^{\alpha}\right) \neq \mathcal{T}_{-e_{x}} \cap B\left((r, 0), r^{\alpha}\right)\right) \\
& =\mathbb{P}\left(\bigcup_{X \in N \cap B\left((r, 0), r^{\alpha}\right)}\left\{\mathcal{A}(X) \neq \mathcal{A}_{-e_{x}}(X)\right\}\right) \\
& \leq \mathbb{P}\left(N\left(B\left((r, 0), r^{\alpha}\right)\right)>r^{\beta}\right)+r^{\beta} C \sup _{X \in N \cap B\left((r, 0), r^{\alpha}\right)} \mathbb{P}\left(\mathcal{A}(X) \neq \mathcal{A}_{-e_{x}}(X)\right) \\
& \leq \exp \left(-r^{\beta} \log \left(\frac{r^{\beta-2 \alpha}}{\mathrm{e} \pi}\right)\right)+r^{\beta} C \frac{r^{\alpha}+1}{r-r^{\alpha}},
\end{aligned}
$$

by using [15, Lemma 11.1.1] for the first term on the right-hand side and [2, Lemma 3.4] for the second term. The first term converges to 0 if and only if $\beta>2 \alpha$ and the second term converges to 0 if and only if $\alpha<1$ and $\beta+\alpha<1$. As a consequence, we see that, for any $\alpha<\frac{1}{3}$, we can choose $\beta>\frac{2}{3}$, so that both terms converge to 0 when $r \rightarrow+\infty$.

\section{Semi-infinite paths in a given direction}

In this section we fix a direction $\theta \in[0,2 \pi)$ and are interested in the semi-infinite paths with asymptotic direction $\theta$. Our first result (Section 3.1) refines Theorem 1.1 and states that there a.s. exists a unique semi-infinite path with direction $\theta$. We deduce from this a precise description of the semi-infinite path with direction $\theta$ (Section 3.2).

For the proof, let us introduce further notation. We define as $\mathcal{T}_{X}$ the subtree of $\mathcal{T}$ consisting of $X \neq O$ and all its descendants, i.e. all the vertices of $\mathcal{T}$ that have $X$ in their ancestry. This tree is naturally rooted at $X$. If $\mathcal{T}_{X}$ is unbounded then we can construct two particular semi-infinite paths that we call the rightmost and leftmost semi-infinite paths, $\underline{\gamma}_{X}$ and $\bar{\gamma}_{X}$, of $\mathcal{T}_{X}$. The construction is as follows. Put $X_{0}=X$. Let

$$
K_{0}=\operatorname{card}\left\{Y \in N, \mathcal{A}(Y)=X_{0}, \text { and } \mathcal{T}_{Y} \text { is unbounded }\right\}
$$


be the number children of $X_{0}$ with infinite descendance. Since the number of children of a given vertex is a.s. finite (see [2, Section 3.3.2]) and since $X_{0}$ has infinitely many descendants, $K_{0} \geq 1$ a.s. It is possible to rank the offspring $X_{0}^{1}, \ldots, X_{0}^{K_{0}}$ in increasing order of the oriented angles $\mathcal{A}\left(\widehat{\left.X_{0}\right) X_{0}} X_{0}^{k}\right.$ for $k \in\left\{1, \ldots, K_{0}\right\}$. Define $X_{1}$ as the child of $X_{0}$ corresponding to the largest value of these angles. Iterating this construction, a semi-infinite path $\bar{\gamma}_{X}=\left(X_{n}\right)_{n \in \mathbb{N}}$ rooted at $X$ is built. In the same way, a semi-infinite path $\underline{\gamma}_{X}$ rooted at $X$ is constructed such that, among the semi-infinite paths of $\mathcal{T}_{X}, \underline{\gamma}_{X}$ is the lowest path (in the trigonometric sense). Consequently, any given semi-infinite path in $\mathcal{T}_{X}$ is trapped between $\underline{\gamma}_{X}$ and $\bar{\gamma}_{X}$ (in the trigonometric sense).

\subsection{Uniqueness}

Part (iii) of Theorem 1.1 ensures the existence of random directions with at least two semiinfinite paths. However, there is no more than one semi-infinite path with a deterministic direction (Proposition 3.1). This result completes part (ii) of Theorem 1.1.

Proposition 3.1. For all $\theta \in[0,2 \pi)$, there a.s. exists exactly one semi-infinite path with asymptotic direction $\theta$ in the RST.

The idea of the proof of Proposition 3.1 is classical; see [10] for first passage percolation models defined from homogeneous PPPs on $\mathbb{R}^{2}$ and [8] for a directed last passage percolation model on the lattice $\mathbb{Z}^{2}$. Thanks to Fubini's theorem, it follows that, for Lebesgue almost every $\theta$ in $[0,2 \pi)$, there is at most one semi-infinite path with asymptotic direction $\theta$ with probability 1 . Actually, this statement holds for all $\theta \in[0,2 \pi)$ by the isotropic character of the PPP $N$.

Proof of Proposition 3.1. Let us denote by $U(\theta)$ the event that there exist at least two different semi-infinite paths in the RST with asymptotic direction $\theta$. Now, assume that the event $U(\theta)$ occurs, and let $\gamma_{1}$ and $\gamma_{2}$ be two such semi-infinite paths. Let $X$ be a point of the PPP $N$ belonging to $\gamma_{1}$ but not to $\gamma_{2}$. Thus, the semi-infinite subpath of $\gamma_{1}$ rooted at $X$ belongs to $\mathcal{T}_{X}$. Then one of the two semi-infinite paths $\underline{\gamma}_{X}$ and $\bar{\gamma}_{X}$ is trapped between $\gamma_{1}$ and $\gamma_{2}$, by planarity and since paths are nonintersecting (see Lemma A.1 in Appendix A). So, it also admits $\theta$ as an asymptotic direction.

Let us denote by $\lambda$ the Lebesgue measure on $[0,2 \pi)$. We are interested in the Lebesgue measure of the set $\{\theta ; U(\theta)\}$ of directions $\theta \in[0,2 \pi)$ where the event $U(\theta)$ is satisfied. The previous remark implies that

$$
\begin{aligned}
\mathbb{E} \lambda\{\theta ; U(\theta)\}= & \int_{\Omega} \int_{0}^{2 \pi} \mathbf{1}_{U(\theta)}(\omega) \mathrm{d} \theta \mathrm{d} \mathbb{P}(\omega) \\
\leq & \int_{\Omega} \sum_{X \in N(\omega)} \mathbf{1}_{\left\{\mathcal{T}_{X} \text { unbounded }\right\}} \\
& \times \int_{0}^{2 \pi} \mathbf{1}_{\left\{\underline{\gamma}_{X} \text { or } \bar{\gamma}_{X} \text { admits } \theta \text { as an asymptotic direction }\right\}}(\omega) \mathrm{d} \theta \mathrm{d} \mathbb{P}(\omega)
\end{aligned}
$$

( $N$ is a.s. countable). For a given point $X \in N$ and a given $\omega \in \Omega$, the indicator function

$$
\mathbf{1}_{\left\{\underline{\gamma}_{X} \text { or } \bar{\gamma} X \text { admits } \theta \text { as an asymptotic direction }\right\}}(\omega)
$$

is equal to 1 for at most two different angles in $[0,2 \pi)$. Its integral is then equal to 0 . Using the 
Fubini theorem,

$$
\int_{0}^{2 \pi} \mathbb{P}(U(\theta)) \mathrm{d} \theta=\mathbb{E} \lambda\{\theta ; U(\theta)\}=0 .
$$

So, the probability $\mathbb{P}(U(\theta))$ is 0 for Lebesgue almost every $\theta$ in $[0,2 \pi)$. Actually, this is true for every $\theta$ in $[0,2 \pi)$ thanks to the isotropic character of the PPP $N$. Combining this with part (ii) of Theorem 1.1, the announced result follows.

\subsection{Further description of the semi-infinite path with direction 0}

In the rest of this section we discuss some consequences of Proposition 3.1. For any given $\theta \in[0,2 \pi)$, let us denote by $\gamma_{\theta}$ the semi-infinite path of the RST, started at the origin and with asymptotic direction $\theta$. It is a.s. well defined by Proposition 3.1. Since the distribution of the RST is invariant by rotation, we will henceforth assume that $\theta=0$.

Let us recall that $\tilde{\chi}_{r}$ denotes the number of intersection points of $a\left(A_{r}, B_{r}\right)$ with the semiinfinite paths of the RST and that $\tilde{\chi}_{r} \rightarrow 0$ in probability, by (2.1). We have the following result.

Corollary 3.1. It holds that $\lim _{\sup } \rightarrow \infty \tilde{\chi}_{r} \geq 1$ a.s.

Proof. Assume that there exists with positive probability a (random) radius $r_{0}$ such that $\tilde{\chi}_{r}=0$ whenever $r>r_{0}$. Let us work on the set where this event is realized. In this case, no semi-infinite path crosses the abscissa axis after $r_{0}$. Then we can exhibit two semi-infinite paths, say $\gamma$ and $\gamma^{\prime}$, respectively below and above the horizontal axis that satisfy the following property: there is no semi-infinite path in the RST, different from $\gamma$ and $\gamma^{\prime}$, and trapped between them (in the trigonometric sense). Parts (i) and (ii) of Theorem 1.1 force $\gamma$ and $\gamma^{\prime}$ to have the same asymptotic direction, namely 0 . Such a situation never happens by Proposition 3.1. In other words,

$$
\mathbb{P}\left(\limsup _{r \rightarrow \infty} \tilde{\chi}_{r} \geq 1\right)=1 .
$$

This completes the proof.

From vertices of $\gamma_{0}$ (different from $O$ ), some paths (finite or not) emanate, forming an unbounded subtree of the RST $\mathcal{T}$ for which $\gamma_{0}$ can be understood as the spine. The next result describes the skeleton of this subtree.

Let us denote by $V_{\infty}^{+}$and $V_{\infty}^{-}$the sets of points $X \in N \cap \gamma_{0} \backslash\{O\}$ from which (at least) another semi-infinite path emanates, respectively above and below $\gamma_{0}$. Of course, $V_{\infty}^{+}$and $V_{\infty}^{-}$ may have a nonempty intersection.

Corollary 3.2. (i) Almost surely, $V_{\infty}^{+}$and $V_{\infty}^{-}$are of infinite cardinality.

(ii) For $r>0$, let us denote by $D_{r}$ the set of directions $\alpha \in[0,2 \pi)$ with a semi-infinite path starting from a point $X$ in $V_{\infty}^{+} \cup V_{\infty}^{-}$with modulus $|X|>r$. Then there a.s. exist two nonincreasing sequences $\left(\alpha_{r}\right)_{r>0}$ and $\left(\beta_{r}\right)_{r>0}$ of positive random variables such that

$$
\left.D_{r}=\left[-\alpha_{r}, \beta_{r}\right] \quad \text { (modulo } 2 \pi\right) \text { and } \lim _{r \rightarrow+\infty} \alpha_{r}=\lim _{r \rightarrow+\infty} \beta_{r}=0 .
$$

(iii) Let $v_{\infty}^{r}$ be the cardinality of $\left(V_{\infty}^{+} \cup V_{\infty}^{-}\right) \cap B(O, r)$. Then

$$
\lim _{r \rightarrow \infty} \mathbb{E} \frac{v_{\infty}^{r}}{r}=0
$$


The first two assertions of Corollary 3.2 state that an infinite number of unbounded subtrees emanate from the semi-infinite path $\gamma_{0}$. Each covers a whole interval of asymptotic directions whose length tends to 0 as its starting point (on $\gamma_{0}$ ) is far from the origin. The last assertion of Corollary 3.2 can be understood as follows: the expected density of points of $\gamma_{0}$ from which emanates another semi-infinite path is 0 . For this purpose, recall that the cardinality of $\gamma_{0} \cap B(O, r)$ is of order $r$ (see [2, Theorem 2.5]).

Proof of Corollary 3.2. On the event $\left\{V_{\infty}^{+}\right.$is finite $\}$, let us consider the point $Y$ of $V_{\infty}^{+}$of highest modulus. Let $Y^{\prime \prime}$ be the child of $Y$ belonging to $\gamma_{0}$. From the definition of $V_{\infty}^{+}$, the set of points $\left\{X \in N, \mathcal{A}(X)=Y, \widehat{Y^{\prime \prime} Y X}>0\right.$, and $\mathcal{T}_{X}$ is unbounded $\}$ is nonempty. The points of this set can be ranked in increasing order of the angles $\widehat{Y^{\prime \prime} Y X}$. Let $Y^{\prime}$ be the point of this set corresponding to the smallest positive angle $\widehat{Y^{\prime \prime} Y X}$. In the subtree $\mathcal{T}_{Y^{\prime}}$, we can define the lowest semi-infinite path $\underline{\gamma}_{Y^{\prime}}$, as defined at the beginning of Section 3. Thanks to part (i) of Theorem 1.1, $\underline{\gamma}_{Y^{\prime}}$ has an asymptotic direction, say $\theta$. Moreover, using the planarity and the noncrossing property of paths together with the definitions of the points $Y$ and $Y^{\prime}$, all the paths between $\gamma_{0}$ and $\underline{\gamma}_{Y^{\prime}}$ (in the trigonometric sense) are finite. Using part (ii) of Theorem 1.1, we deduce that $\underline{\gamma}_{Y^{\prime}}$ and $\gamma_{0}$ have the same asymptotic direction, i.e. $\theta=0$. Now, by Proposition 3.1, such a situation never occurs. So the set $V_{\infty}^{+}$is a.s. infinite. The same goes for $V_{\infty}^{-}$.

Let us prove part (ii) of Corollary 3.2. For any $r$, consider the point $X$ of smallest modulus among the points of $\gamma_{0} \cap B(O, r)^{\mathrm{c}}$. The semi-infinite paths $\underline{\gamma}_{X}$ and $\bar{\gamma}_{X}$ a.s. have asymptotic directions, say respectively $-\alpha_{r}$ and $\beta_{r}$ (modulo $2 \pi$ ) with $\alpha_{r} \geq 0$ and $\beta_{r} \geq 0$. Hence, $\left(\alpha_{r}\right)_{r>0}$ and $\left(\beta_{r}\right)_{r>0}$ are by construction nonincreasing sequences of positive real numbers (by Proposition 3.1).

Let us consider $D_{r}$, the set of directions corresponding to semi-infinite paths starting from points in $V_{\infty}^{+} \cup V_{\infty}^{-}$with modulus greater than $r$. The set $D_{r}$ contains $\alpha_{r}$ and $\beta_{r}$ defined above. The bi-infinite path obtained by concatenation of $\underline{\gamma}_{X}$ and $\bar{\gamma}_{X}$ divides $\mathbb{R}^{2}$ into two unbounded regions. Since the paths of the RST cannot cross, $\bar{D}_{r}^{X}$ is included in the real interval $\left[-\alpha_{r}, \beta_{r}\right]$. This also forces any given semi-infinite path with asymptotic direction $\alpha$ in $\left[-\alpha_{r}, \beta_{r}\right]$ to go through the vertex $X$. By part (ii) of Theorem 1.1, $D_{r}$ is then an interval. It follows that $D_{r}=\left[-\alpha_{r}, \beta_{r}\right]$.

Finally, let us respectively denote by $\bar{\alpha}$ and $\bar{\beta}$ the limits of sequences $\left(\alpha_{r}\right)_{r>0}$ and $\left(\beta_{r}\right)_{r>0}$. Let $\beta>0$. Let $X_{0, \beta}$ be the bifurcation point of $\gamma_{0}$ and the semi-infinite path with asymptotic direction $\beta$ (whose existence and uniqueness are given by Proposition 3.1). Then $\bar{\beta} \geq \beta$ implies that $\beta$ belongs to any interval $D_{r}$. In other words,

$$
\mathbb{P}(\bar{\beta} \geq \beta)=\lim _{r \rightarrow \infty} \mathbb{P}\left(\left|X_{0, \beta}\right|>r\right)=0 .
$$

As a consequence, $\bar{\beta}$ is a.s. equal to 0 . Similarly, we can prove that $\bar{\alpha}=0$.

Part (iii) of Corollary 3.2 follows directly from the inequality $v_{\infty}^{r} \leq \chi_{r}$, where $\chi_{r}$ counts the intersection points of $S(O, r)$ with the semi-infinite paths of the RST, and Theorem 2.1.

\section{The colored RST}

The aim of this section is to describe the subtrees of the RST $\mathcal{T}$ rooted at the children of $O$. In order to distinguish them, let us start by allocating a color or a label (denoted by integers $i, j, k, \ldots$ ) to each child $X$ of $O$. Recall that, for this purpose, there are a.s. at most five (see Lemma 3.2 of [2]). Then we paint all the vertices of the subtrees $\mathcal{T}_{X}$ with the color of $X$. This process provides a coloration of points of $N \backslash\{O\}$. Finally, each segment $[X, \mathcal{A}(X)]$ for 
(a)

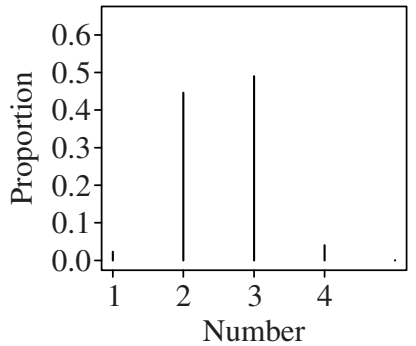

(d)

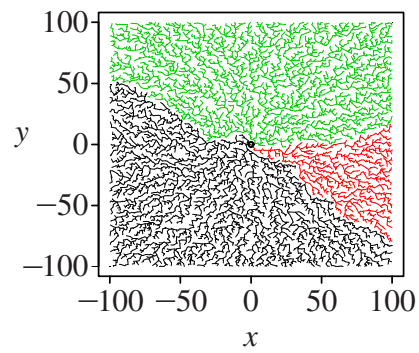

(b)

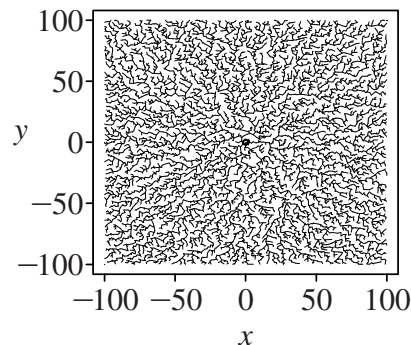

(e)

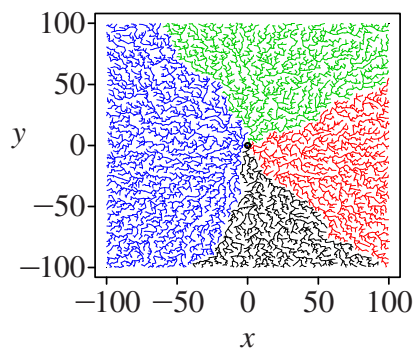

(c)

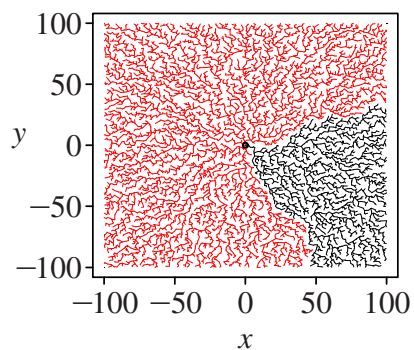

(f)

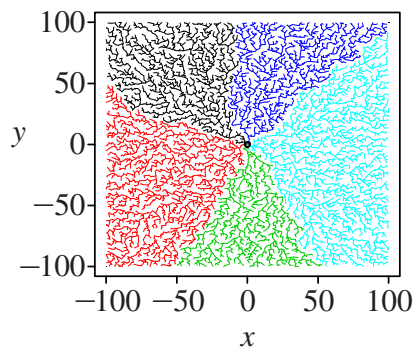

FIGURE 3: (a) Empirical distribution for the number of children of $O$. Over 5000 simulations, 1 (respectively 2, 3, 4, and 5) child is obtained in 114 (respectively 2232, 2449, 203, and 2) cases. Simulations of $m=1, \ldots, 5$ subtrees of the RST rooted at the children of $O$ are given in (b) to (f). They seem to be unbounded.

$X \in N \backslash\{O\}$ is painted with the color of $X$. This can be done without ambiguity thanks to the planarity and the noncrossing property of paths. See Figure 3.

There are several ways to label the subtrees of $\mathcal{T}$ rooted at $O$ with the colors. We use the notation $i, j$, etc. when the labeling is not important and can be any labeling (one possibility is to start from the angle 0 (abscissa axis) and label by 1 the subtree rooted at the first descendant of $O$ that we encounter when exploring the directions in the trigonometric sense). However, to take advantage of the exchangeability of the different colored trees, one may also proceed as follows. To each of the direct descendants of $O$, a uniform independent random variable is attached. We then define the tree with color 1 as the tree consisting of the offspring of the descendant with the smallest uniform random variable. This amounts to choosing one of the descendants at random for the first tree. When we proceed so, we use the notation $1, \ldots, \underline{i}, \ldots$ for the labels.

The next step is to define the competition interfaces, i.e. the borders between the subtrees of the RST rooted at the children of the origin. To do so, let us introduce the spatially embedded version of the RST $\mathcal{T}$, denoted by $\mathbb{T}$, as the following subset of $\mathbb{R}^{2}$ :

$$
\mathbb{T}=\bigcup_{X \in N \backslash\{O\}}[X, \mathcal{A}(X)] .
$$

For any positive real number $r$, the normalized trace of $\mathbb{T}$ over the sphere $S(O, r)$ is

$$
\mathbb{T}_{r}=\frac{1}{r}(\mathbb{T} \cap S(O, r)) .
$$


An element $u$ of $\mathbb{T}_{r}$ inherits its color from the element $r u \in \mathbb{T}$. So, for any given color $i$, we denote by $\mathbb{T}_{r}(i)$ the points of $\mathbb{T}_{r}$ with color $i$. By the noncrossing paths property of the RST, the points of $\mathbb{T}_{r}$ are 'gathered' on the unit sphere $S(O, 1)$ according to their color. This can be formalized as follows: for any $r>0, \theta_{1}, \theta_{2}, \theta_{3}, \theta_{4} \in[0,2 \pi)$ such that $\left(\theta_{1}-\theta_{3}\right)\left(\theta_{2}-\theta_{3}\right)>0$, $\left(\theta_{1}-\theta_{4}\right)\left(\theta_{2}-\theta_{4}\right)<0, \mathrm{e}^{\mathrm{i} \theta_{1}}, \mathrm{e}^{\mathrm{i} \theta_{2}} \in \mathbb{T}_{r}(i)$, and $\mathrm{e}^{\mathrm{i} \theta_{3}}, \mathrm{e}^{\mathrm{i} \theta_{4}} \in \mathbb{T}_{r}$, at least one of the two points $\mathrm{e}^{\mathrm{i} \theta_{3}}$ and $\mathrm{e}^{\mathrm{i} \theta_{4}}$ is of color $i$.

For all $\left(\theta, \theta^{\prime}\right) \in[0,2 \pi)^{2}$, let us denote by $a\left(\theta, \theta^{\prime}\right)$ and $\bar{a}\left(\theta, \theta^{\prime}\right)$ the arcs of the unit sphere from $\mathrm{e}^{\mathrm{i} \theta}$ to $\mathrm{e}^{\mathrm{i} \theta^{\prime}}$ in the trigonometric sense, respectively without and with the endpoints $\mathrm{e}^{\mathrm{i} \theta}$ and $\mathrm{e}^{\mathrm{i} \theta^{\prime}}$. Furthermore, let $\mathbb{T}(i)$ be the subset of $\mathbb{T}$ with color $i$.

Definition 4.1. (Competition interfaces.) Given a couple of colors $(i, j)$ with $i \neq j$, there exists at most one couple $\left(\theta, \theta^{\prime}\right) \in[0,2 \pi)^{2}$ such that

$$
\mathrm{e}^{\mathrm{i} \theta} \in \mathbb{T}_{r}(i), \quad \mathrm{e}^{\mathrm{i} \theta^{\prime}} \in \mathbb{T}_{r}(j), \quad \text { and } \quad a\left(\theta, \theta^{\prime}\right) \cap \mathbb{T}_{r}=\varnothing .
$$

When such a couple $\left(\theta, \theta^{\prime}\right)$ exists, we denote by $\theta_{r}(i, j) \in[0,2 \pi)$ the (direct) angle of the line coming from $O$ and bisecting the arc $a\left(\theta, \theta^{\prime}\right)$ in two equal parts (see Figure 4). In this case, the competition interface between the sets $\mathbb{T}(i)$ and $\mathbb{T}(j)$ is defined as the curve

$$
\varphi(i, j)=\left\{r \mathrm{e}^{\mathrm{i} \theta_{r}(i, j)} \in \mathbb{C}, \beta(i, j)<r<\partial(i, j)\right\},
$$

where $\beta(i, j)$ and $\partial(i, j)$ are respectively defined as the infimum and the supremum of the set $\left\{r>0, \theta_{r}(i, j)\right.$ exists $\}$.

From $\beta(i, j)$ to $\partial(i, j)$, the trees $\mathbb{T}(i)$ and $\mathbb{T}(j)$ evolve in the plane side by side, separated by the competition interface $\varphi(i, j)$. The real numbers $\beta(i, j)$ and $\partial(i, j)$ can respectively be interpreted as the birth and death times of the competition interface $\varphi(i, j)$. When $\partial(i, j)=$ $+\infty$, both sets $\mathbb{T}(i)$ and $\mathbb{T}(j)$ are unbounded. When $\partial(i, j)<+\infty$, one of the two sets $\mathbb{T}(i)$ and $\mathbb{T}(j)$ is included in the closed ball $\bar{B}(O, \partial(i, j))$, say $\mathbb{T}(j)$. In this case, $\partial(i, j)$ coincides with another death time $\partial(j, k)$ and two situations may occur according to the color $k$. Either $k=i$,

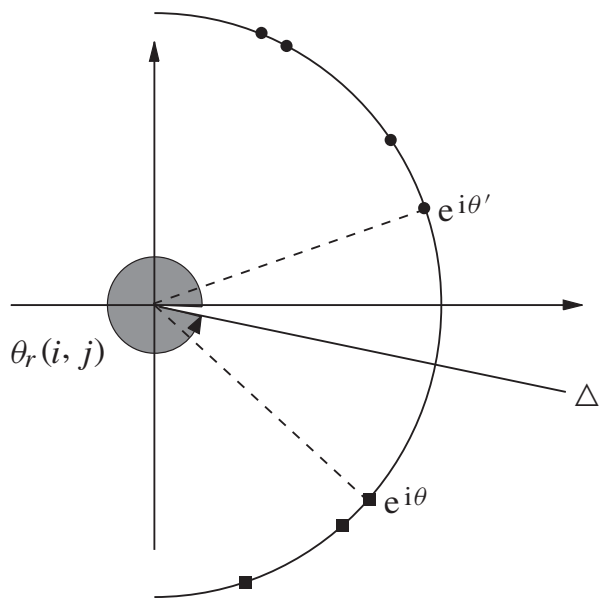

FIGURE 4: On the unit sphere, the black squares are the points of $\mathbb{T}_{r}(i)$ and the black circles are the points of $\mathbb{T}_{r}(j)$. The arc $a\left(\theta, \theta^{\prime}\right)$ is divided into two equal parts by the line $\Delta$ whose angle (represented in gray) is $\theta_{r}(i, j)$. 
which means that $i$ is the only existing color outside the ball $\bar{B}(O, \partial(i, j))$ and there is no competition interface beyond that ball, or $k$ is a third color (different from $i$ and $j$ ). Then the competition interface $\varphi(i, k)$ extends $\varphi(i, j)$ and $\varphi(j, k)$ (until its death time $\partial(k, j)$ ). Its birth time satisfies

$$
\beta(i, k)=\partial(i, j)=\partial(j, k)>0 .
$$

Let us remark that the application $r \mapsto \theta_{r}(i, j)$ may be discontinuous. Finally, note that $\theta_{r}(i, j) \neq \theta_{r}(j, i)$ and that one may exist and the other not. So, we distinguish the interfaces $\varphi(i, j)$ and $\varphi(j, i)$.

Our first result below states that there can be up to five unbounded competition interfaces with positive probability. See Figure 5 and Table 1 for empirical results.

Theorem 4.1. For any $m \in\{1,2,3,4,5\}$, there exist (exactly) $m$ unbounded subtrees of $\mathcal{T}$ with different colors, with positive probability. In other words, for any $m \in\{0,2,3,4,5\}$, there exist (exactly) $m$ unbounded competition interfaces, with positive probability.

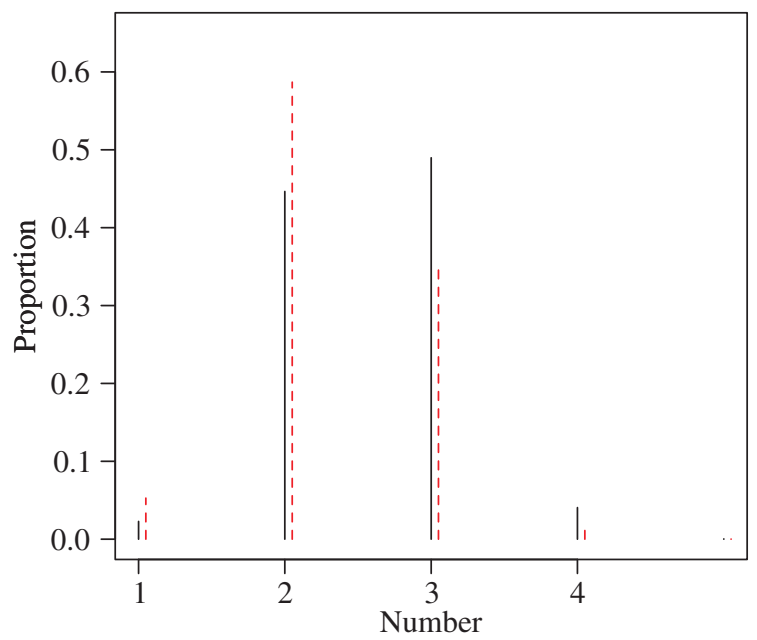

FIGURE 5: (a) Empirical distributions, obtained after $N=5000$ simulations, for the number of children of $O$ (solid lines) and for the number of unbounded subtrees (dashed lines). Percentages are given in Table 1.

TABLE 1: The percentages relating to the empirical distributions of Figure 5. The two distributions are different since the tree associated with each child of $O$ is not necessarily unbounded. Note that the cases $m \in\{4,5\}$ are very rare (less than $2 \%$ of the simulations) compared with the cases $m \in\{2,3\}$ (more than $93 \%$ ). Actually, configurations corresponding to $m \in\{4,5\}$ are very constrained around the origin and, therefore, rare.

\begin{tabular}{ccc}
\hline$m$ & Children of $O$ & Unbounded subtrees \\
\hline 1 & 2.28 & 5.28 \\
2 & 44.64 & 58.68 \\
3 & 48.98 & 34.64 \\
4 & 4.06 & 1.38 \\
5 & 0.04 & 0.02 \\
\hline
\end{tabular}


Our proof relies on part (i) of Theorem 1.1. Thinning and local modification of the PPP are other ingredients.

Proof of Theorem 4.1. We consider the cases $m \in\{1, \ldots, 5\}$ separately.

Case 1: $m=5$. Our purpose is to construct a set of configurations of $N$, with a positive probability, on which there are five children of the origin $O$ giving birth to infinite subtrees.

For any $1 \leq k \leq 5$, part (i) of Theorem 1.1 ensures the almost-sure existence of a semiinfinite path $\gamma_{k}$ with asymptotic direction $2 k \pi / 5$. Hence, for $\varepsilon>0$ and with probability 1 , there exists a (random) radius $r_{k}$ such that $\gamma_{k}$ is included in the cone section

$$
C_{2 k \pi / 5, \varepsilon, r_{k}}=\left\{\rho \mathrm{e}^{\mathrm{i} \theta} ; \rho>r_{k} \text { and }\left|\theta-\frac{2 k \pi}{5}\right|<\varepsilon\right\}
$$

for any integer $1 \leq k \leq 5$. Without loss of generality, we can require that $\gamma_{k}$ starts from a vertex $X_{k} \in N$ whose norm satisfies $r_{k}<\left|X_{k}\right| \leq r_{k}+1$ and for $r_{k}$ to be a positive integer. Hence, writing

$$
\begin{aligned}
A_{\varepsilon}\left(r_{1}, \ldots, r_{5}\right)= & \left\{\text { for any } 1 \leq k \leq 5, \text { there exists a semi-infinite path } \gamma_{k}\right. \\
& \text { included in the cone } C_{2 k \pi / 5, \varepsilon, r_{k}} \text { and starting from a } \\
& \text { vertex } \left.X_{k} \text { satisfying } r_{k}<\left|X_{k}\right| \leq r_{k}+1\right\},
\end{aligned}
$$

we find that, for all $\varepsilon>0$, there exist some (deterministic) radii $r_{1}, \ldots, r_{5} \in \mathbb{N}^{*}$ such that $A_{\varepsilon}\left(r_{1}, \ldots, r_{5}\right)$ occurs with positive probability.

Let $R=\max \left\{r_{k}+1 ; 1 \leq k \leq 5\right\}$ and $V_{\varepsilon}\left(r_{1}, \ldots, r_{5}\right)$ be the complementary set of the five cones in the ball $B(O, R)$ :

$$
V_{\varepsilon}\left(r_{1}, \ldots, r_{5}\right)=B(O, R) \backslash\left[\left(\bigcup_{k=1}^{5} C_{2 k \pi / 5, \varepsilon, r_{k}}\right) \cup\{O\}\right] .
$$

Now, we are going to change the configuration of the PPP $N$ in $V_{\varepsilon}\left(r_{1}, \ldots, r_{5}\right)$ in such a way that the $X_{k}$ are all different colors. Let $\tilde{N}=N \cap V_{\varepsilon}^{\mathrm{c}}\left(r_{1}, \ldots, r_{5}\right)$ be the thinned PPP obtained by deleting all the points of $N$ belonging to $V_{\varepsilon}\left(r_{1}, \ldots, r_{5}\right)$ (see, e.g. [12, Section II.4.b]). It is crucial to note that deleting the points of $V_{\varepsilon}\left(r_{1}, \ldots, r_{5}\right)$ does not affect the occurrence of $A_{\varepsilon}\left(r_{1}, \ldots, r_{5}\right)$. In other words, if $N$ satisfies the event $A_{\varepsilon}\left(r_{1}, \ldots, r_{5}\right)$, so does $\tilde{N}$ :

$$
\mathbb{P}\left(\tilde{N} \in A_{\varepsilon}\left(r_{1}, \ldots, r_{5}\right)\right) \geq \mathbb{P}\left(N \in A_{\varepsilon}\left(r_{1}, \ldots, r_{5}\right)\right)>0 .
$$

Now let us consider a PPP $\hat{N}$ on $V_{\varepsilon}\left(r_{1}, \ldots, r_{5}\right)$ with intensity 1 . Let us denote by $r$ the minimum of the $r_{k}$.

The event $\hat{N} \in B_{\varepsilon}\left(r_{1}, \ldots, r_{5}\right)$ is defined by the three following conditions.

(C1) For any $k \in\{1, \ldots, 5\}$, if $r_{k}>r$ then, for all integers $r \leq n \leq r_{k}-1$,

$$
\hat{N}\left(B\left(n \mathrm{e}^{\mathrm{i} 2 k \pi / 5}, \varepsilon\right)\right)=1 ;
$$

otherwise,

$$
\hat{N}\left(B\left(r \mathrm{e}^{\mathrm{i} 2 k \pi / 5}, \varepsilon\right) \cap B(O, r)\right)=1 .
$$

(C2) For any $k \in\{1, \ldots, 5\}$ and all integers $n$ such that $0 \leq n \leq\left(R-r_{k}-1\right) / 2 \varepsilon$,

$$
\hat{N}\left(B\left(\left(r_{k}+1+2 n \varepsilon\right) \mathrm{e}^{\mathrm{i}(2 k \pi / 5 \pm 2 \varepsilon)}, \varepsilon\right) \cap V_{\varepsilon}\left(r_{1}, \ldots, r_{5}\right)\right)=1 .
$$

(C3) The previous points are the only points of $\hat{N}$. 


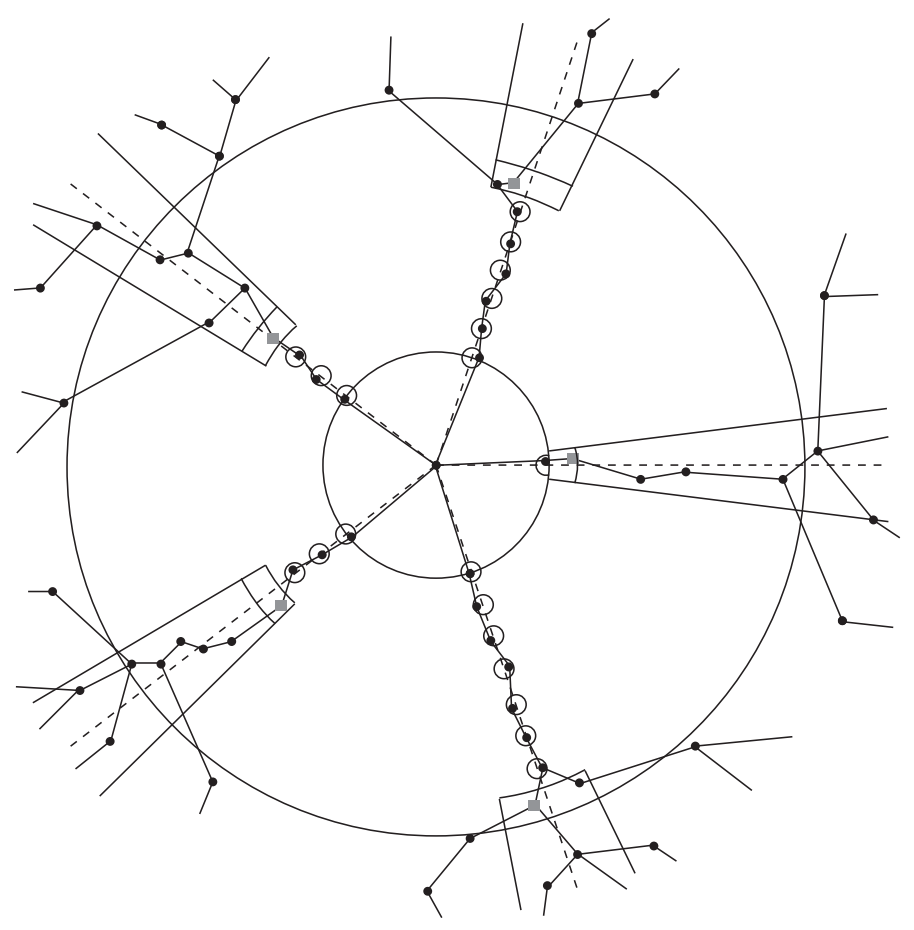

FIGURE 6: RST of the PPP $N$ satisfying both events $A_{\varepsilon}\left(r_{1}, \ldots, r_{5}\right)$ and $B_{\varepsilon}\left(r_{1}, \ldots, r_{5}\right)$. Note that, in order not to overload the figure, condition (C2) of $B_{\varepsilon}\left(r_{1}, \ldots, r_{5}\right)$ has not been represented. The two balls are centered at $O$ with radii $r=\min _{k \in\{1, \ldots, 5\}} r_{k}$ and $R=\max _{k \in\{1, \ldots, 5\}} r_{k}+1$. The $X_{k}$ are represented by gray squares, while the other points of $N$ are represented by black circles.

It is clear that the event $\hat{N} \in B_{\varepsilon}\left(r_{1}, \ldots, r_{5}\right)$ occurs with positive probability for all $\varepsilon>0$. Roughly speaking, the points of $\hat{N}$ introduced in (C1) form a chain from $r \mathrm{e}^{\mathrm{i} 2 k \pi / 5}$ to $\left(r_{k}-1\right) \mathrm{e}^{\mathrm{i} 2 k \pi / 5}$ for any index $k$ such that $r_{k}>r$. See Figure 6 .

For Figure 6, imagine that $R=r_{4}+1$ is much larger than $r=r_{5}$ (indeed, we have no control over the $r_{k}$ ). Henceforth, the semi-infinite path $\gamma_{5}$ could prefer to branch on the points of $\hat{N}$ introduced in $(\mathrm{C} 1)$ and with direction $8 \pi / 5$ rather than on $X_{5}$. To prevent this situation from occurring, we contain each path $\gamma_{k}$ in the cone $C_{2 k \pi / 5,3 \varepsilon, r_{k}}$ thanks to the points of $\hat{N}$ introduced in (C2). These points form 'landing runways' for the $\gamma_{k}$ (they may also slightly change the $\gamma_{k}$ ).

Let us denote by $A_{\varepsilon}$ and $B_{\varepsilon}$ the events $A_{\varepsilon}\left(r_{1}, \ldots, r_{5}\right)$ and $B_{\varepsilon}\left(r_{1}, \ldots, r_{5}\right)$. Then

$$
\left\{\tilde{N} \in A_{\varepsilon}\right\} \cap\left\{\hat{N} \in B_{\varepsilon}\right\} \subset\left\{\tilde{N}+\hat{N} \in A_{3 \varepsilon} \cap B_{\varepsilon}\right\},
$$

where $\tilde{N}+\hat{N}$ denotes the superposition of the two processes $\hat{N}$ and $\tilde{N}$. These two processes can also be assumed independent. In this case, $\tilde{N}+\hat{N}$ is still a PPP on $\mathbb{R}^{2}$. It follows that

$$
\begin{aligned}
\mathbb{P}\left(N \in A_{3 \varepsilon} \cap B_{\varepsilon}\right) & =\mathbb{P}\left(\tilde{N}+\hat{N} \in A_{3 \varepsilon} \cap B_{\varepsilon}\right) \\
& \geq \mathbb{P}\left(\tilde{N} \in A_{\varepsilon}, \hat{N} \in B_{\varepsilon}\right) \\
& \geq \mathbb{P}\left(\tilde{N} \in A_{\varepsilon}\right) \mathbb{P}\left(\hat{N} \in B_{\varepsilon}\right) \\
& >0 .
\end{aligned}
$$


To conclude the proof, it remains to prove that the above event implies the existence of (at least) five unbounded subtrees of $\mathcal{T}$ with different colors. Actually, there will be exactly five unbounded subtrees since the degree of $O$ is a.s. upper bounded by 5 . Let us denote by $Y_{k}$ the point of $N$ belonging to the ball $B\left(r \mathrm{e}^{\mathrm{i} 2 k \pi / 5}, \varepsilon\right)$. On the event $N \in A_{3 \varepsilon} \cap B_{\varepsilon}$, the point $X_{k}$ is a descendant of $Y_{k}$ for any $k$. Hence, the subtrees rooted at $Y_{1}, \ldots, Y_{5}$ are unbounded. Finally, it suffices to note that the $Y_{k}$ have $O$ as a common ancestor. Indeed, each $Y_{k}$ is at a distance smaller than $\varepsilon$ from $\mathrm{e}^{\mathrm{i} 2 k \pi / 5}$. So,

$$
\left|Y_{k+1}-Y_{k}\right| \geq\left|r \mathrm{e}^{\mathrm{i} 2(k+1) \pi / 5}-r \mathrm{e}^{\mathrm{i} 2 k \pi / 5}\right|-2 \varepsilon \geq 2 r \sin \left(\frac{\pi}{5}\right)-2 \varepsilon \geq 1.17 r-2 \varepsilon,
$$

which is larger than the maximal distance between $Y_{k}$ and $O$, i.e. $r+\varepsilon$ for small enough $\varepsilon$ (using $r \in \mathbb{N}^{*}$ ).

Case 2: $m \in\{3,4\}$. The previous construction applied to $m \in\{3,4\}$ allows us to state that, with positive probability, the origin $O$ has at least $m$ descendants from which $m$ unbounded trees arise. Now, so as to ensure the number of unbounded subtrees of different colors is exactly $m$, an additional precaution must be taken. More precisely, the following condition is added to the event $\hat{N} \in B_{\varepsilon}$.

(C4) For any $k \in\{1, \ldots, m\}$, the argument of the point $Y_{k}$ of $\hat{N} \cap B\left(r \mathrm{e}^{\mathrm{i} 2 k \pi / m}, \varepsilon\right)$ belongs to $(2 k \pi / m-\varepsilon, 2 k \pi / m)$.

Thanks to (C4), each sector of the ball $B(O, r+1)$ with angle $2 \pi / m$ contains (at least) one of the points $Y_{1}, \ldots Y_{m}$. Assume that $N \in A_{3 \varepsilon} \cap B_{\varepsilon}$, which still occurs with positive probability. By construction, the origin $O$ has exactly $m$ children in the ball $B(O, R)$. Let us consider a point $X \in N \backslash\left\{O, Y_{1}, \ldots, Y_{m}\right\}$ such that $|X| \geq R \geq r+1$. Then $B(O,|X|) \cap B(X,|X|)$ contains a sector of the ball $B(O, r+1)$ with angle $2 \pi / 3$, and so contains one of $Y_{1}, \ldots, Y_{m}$. The origin $O$ cannot be the ancestor of $X$. This proves that $O$ is exactly of degree $m$ and completes the proof for $m \in\{3,4\}$.

This latter argument no longer works when $m$ is equal to 1 or 2 .

Case 3: $m=2$. Following the construction for $m=5$, there exists $r_{1}$ and $r_{2}>0$ such that there exist with positive probability two semi-infinite paths $\gamma_{1}$ and $\gamma_{2}$ included in the cones $C_{0, \varepsilon, r_{1}}$ and $C_{\pi, \varepsilon, r_{2}}$. The following event has a positive probability.

- For a given increasing subsequence $\left(\theta_{j}\right)_{j \in \mathbb{N}}$ of $[0, \pi)$ with a sufficiently small step, and for a sufficiently small $\varepsilon>0$,

$$
N\left(B\left(\left(r_{1} \wedge r_{2}\right)\left(1+\cos \left(\theta_{j}\right)\right) \mathrm{e}^{\mathrm{i} \theta_{j}}, \varepsilon\right)\right)=1, \quad N\left(B\left(-\left(r_{1} \wedge r_{2}\right)\left(1+\cos \left(\theta_{j}\right)\right) \mathrm{e}^{\mathrm{i} \theta_{j}}, \varepsilon\right)\right)=1 .
$$

- For all integers $n$ and $m$ such that $0 \leq n \leq\left(r_{2}-r_{1}\right) / 2 \varepsilon$ and $0 \leq n \leq\left(r_{1}-r_{2}\right) / 2 \varepsilon$, if they exist,

$$
N\left(B\left(\left(r_{1}+2 n \varepsilon, 0\right), \varepsilon\right)\right)=1, \quad N\left(B\left(\left(0, r_{2}-2 n \varepsilon\right), \varepsilon\right)\right)=1 .
$$

- The rest of $B\left(O, r_{1} \vee r_{2}\right)$ is empty.

The idea is that in $B\left(O, r_{1} \wedge r_{2}\right)$ the points are roughly aligned following the reunion of two cardiods $\{\rho(\theta)= \pm(1+\cos (\theta)), \theta \in[0, \pi)\}$. Note that this curve is differentiable at $O$ with a horizontal tangent. If $r_{1}<r_{2}$, we add points along the line segment $\left[\left(r_{1}, 0\right),\left(r_{2}, 0\right)\right]$. If the $\theta_{j}$ define a sufficiently fine subdivision of $[0, \pi)$ then there cannot be more than two descendants of $O$ by construction. We conclude as in the $m=5$ case. 
We note that the two semi-infinite paths previously built have asymptotic directions opposed to the argument of the descendant of $O$ from which they stem.

Case 4: $m=1$. Since the RST $\mathcal{T}$ is unbounded, it suffices to prove that the origin $O$ may have only one child with positive probability.

From $z_{1}=\mathrm{e}^{\mathrm{i} \pi / 3}$, we build five complex numbers $z_{2}, \ldots, z_{6}$ by induction: for $k \geq 2$, $z_{k}=\left|z_{k}\right| \mathrm{e}^{\mathrm{i} k \pi / 3}$, whose modulus $\left|z_{k}\right|$ is such that $\left|z_{k}-z_{k-1}\right|<\left|z_{k}\right|$. This construction forces $\left|z_{k}\right|>\left|z_{k-1}\right|$. Let $\varepsilon>0$ be small enough such that $\left|z_{k}\right|-\varepsilon>\left|z_{k-1}\right|+\varepsilon$. Hence, the six balls $B\left(z_{1}, \varepsilon\right), \ldots, B\left(z_{6}, \varepsilon\right)$ do not overlap. Let $\Omega_{\varepsilon}$ be the event that, for all $1 \leq k \leq 6$,

$$
N\left(B\left(z_{k}, \varepsilon\right)\right)=1 \quad \text { and } \quad N\left(B\left(O,\left|z_{6}\right|+\varepsilon\right)\right)=7
$$

(these seven points including the origin). For all $\varepsilon>0, \mathbb{P}\left(\Omega_{\varepsilon}\right)>0$. So, it remains to choose $\varepsilon>0$ small enough in order to ensure that, on the event $\Omega_{\varepsilon}$, the origin $O$ has only one child.

Let us denote by $X_{k}$ the point of $N \cap B\left(z_{k}, \varepsilon\right)$. Since $N \cap B\left(O,\left|X_{1}\right|\right)$ is reduced to $O$, the ancestor of $X_{1}$ is the origin $O$. Thus, for $2 \leq k \leq 6$, we can choose $\varepsilon$ such that

$$
\left|X_{k}-X_{k-1}\right| \leq\left|z_{k}-z_{k-1}\right|+2 \varepsilon<\left|z_{k}-O\right|-\varepsilon \leq\left|X_{k}-O\right| .
$$

This condition does not prove that $X_{k-1}$ is the ancestor of $X_{k}$, but it is not $O$. Finally, let $X$ be a point of the PPP $N$ which does not belong to $B\left(O,\left|z_{6}\right|+\varepsilon\right)$. The set $B(X,|X|) \cap B(O,|X|)$ contains an angular sector of the ball $B\left(O,\left|z_{6}\right|+\varepsilon\right)$ with central angle $2 \pi / 3$. So, it also contains one of the $X_{k}$, preventing $X$ from being a child of $O$. To sum up, $X_{1}$ is the only child of the origin $O$. This completes the proof of Theorem 4.1 .

Let $\Omega(i, j)$ be the event corresponding to an unbounded competition interface $\varphi(i, j)$. It occurs with a positive probability thanks to Theorem 4.1. Recall that $\varphi(i, j)$ separates the two colored subtrees $\mathbb{T}(i)$ and $\mathbb{T}(j)$ in the trigonometric sense. The next result states that $\varphi(i, j)$ a.s. has an asymptotic direction on the event $\Omega(i, j)$. In other words, if $\mathbb{T}(i)$ is unbounded then it asymptotically behaves as a cone.

Proposition 4.1. On the event $\Omega(i, j)$, the sequence $\left(\theta_{r}(i, j)\right)_{r>\beta(i, j)}$ converges a.s. to a random angle $\theta(i, j) \in[0,2 \pi)$.

Proof. Let us consider the event $\Omega(i, j)$ satisfied. Let $X(i)$ and $X(j)$ be the children of the origin of colors $i$ and $j$. On $\Omega(i, j)$, both subtrees $\mathcal{T}_{X(i)}$ and $\mathcal{T}_{X(j)}$ are unbounded. Recall that $\bar{\gamma}_{X(i)}$ denotes the highest (in the trigonometric sense) semi-infinite path in $\mathcal{T}_{X(i)}$ (see the proof of Proposition 3.1). In the same way, $\underline{\gamma}_{X(j)}$ is the lowest semi-infinite path in $\mathcal{T}_{X(j)}$. On $\Omega(i, j)$, the region delimited by $\bar{\gamma}_{X(i)}$ and $\underline{\bar{\gamma}}_{X(j)}^{X(j)}$ (in the trigonometric sense) contains only finite paths. It may also contain some vertices of a third color (different from $i$ and $j$ ). Then, by parts (i) and (ii) of Theorem 1.1, $\bar{\gamma}_{X(i)}$ and $\underline{\gamma}_{X(j)}$ have the same asymptotic direction, say $\theta(i, j)$. To conclude, it suffices to note that the competition interface $\varphi(i, j)$ is trapped between $\bar{\gamma}_{X(i)}$ and $\underline{\gamma}_{X(j)}$. It then admits the same direction.

Proposition 4.1 states that every competition interface that separates the colors $i$ and $j$ has an asymptotic direction $\theta(i, j)$. The next proposition gives a result on the distribution of the asymptotic directions, which remains, however, partial. Recall that we use the labels $\underline{1}, \ldots, \underline{i}, \ldots$ when the subtrees rooted at $O$ are labeled randomly. If the marginal distributions of the $\theta(\underline{i}, i+1)$ are easy to obtain, this is not the case for the distributions of the $\theta(i, i+1)$ which necessitate knowledge of the joint distributions of the asymptotic directions (or, equivalently, the distribution of the sectors between the competition interfaces). In Section 5 we provide numerical simulations and conjectures. 
Proposition 4.2. Conditionally on having $m \geq 2$ infinite trees, and when the tree with color 1 is drawn randomly, the asymptotic directions $\theta(\underline{i}, \underline{i+1})$ are uniformly distributed on $[0,2 \pi)$. Moreover, the distribution of $\theta(i, j)$, on $\Omega(i, j)$, admits a density with respect to the Lebesgue measure on $[0,2 \pi)$.

Proof. Thanks to the rotation invariance property of the PPP and the random labeling $\underline{1}, \ldots, \underline{i}, \ldots$, the distribution of the asymptotic direction $\theta(\underline{i}, \underline{i+1})$ is also invariant by rotation. This property characterizes the Haar probability measure on the circle. The first part of Proposition 4.2 follows.

The event $\{\theta(i, j)=\alpha\}$ implies the existence of at least two semi-infinite paths with the deterministic direction $\alpha$. This is forbidden by Proposition 3.1. So, $\theta(i, j)$ has no atom (when it exists). In fact, the distribution of $\theta(i, j)$ is even absolutely continuous with respect to the Lebesgue measure $\lambda$ on $[0,2 \pi)$. Let $A$ be a measurable subset of $[0,2 \pi)$ such that $\lambda(A)=0$. Let us denote by $M$ the random number of interfaces that exist. Then, for $i \neq j$, since $\theta(i, j)$ corresponds to one of the $\theta(\underline{k}, \underline{l})$ when we relabel the subtrees rooted at $O$ randomly,

$$
\begin{aligned}
& \mathbb{P}(\{\theta(i, j) \in A\} \cap\{M \geq 2\} \cap \Omega(i, j)) \\
& \quad=\sum_{m=2}^{5} \mathbb{P}(\{\theta(i, j) \in A\} \cap\{M=m\} \cap \Omega(i, j)) \\
& \quad \leq \mathbb{P}\left(\bigcup_{i \neq j \in\{1, \ldots, m\}}\{\theta(\underline{i}, \underline{j}) \in A\} \cap\{M=m\} \cap \Omega(\underline{i}, \underline{j})\right) \\
& \quad \leq \sum_{m=2}^{5} \sum_{i \neq j \in\{1, \ldots, m\}} \mathbb{P}(\{\theta(\underline{i}, \underline{j}) \in A\} \cap\{M=m\}) \\
& \quad \leq 0,
\end{aligned}
$$

since $\lambda(A)=0$. The Radon-Nikodym theorem completes the proof.

We conclude this section with a corollary that states that the asymptotic directions of competition interfaces and of semi-infinite paths are related.

Corollary 4.1. The asymptotic direction of the competition interface $\varphi(i, j)$ belongs to the (random) set $D$ of directions with at least two semi-infinite paths. This set is a.s. dense in $[0,2 \pi)$ and countable.

Proof. The fact that $D$ is dense in $[0,2 \pi)$ follows from part (iii) of Theorem 1.1. It is also a.s. countable. Indeed, let us consider the set $\Gamma$ of couples $\left(\gamma_{1}, \gamma_{2}\right)$ of different semi-infinite paths of the RST such that the region they delimit (in the trigonometric sense) contains only finite paths. Associating to each element $\left(\gamma_{1}, \gamma_{2}\right)$ of $\Gamma$ the child in $\gamma_{1}$ of their bifurcation point, we get an injective function from $\Gamma$ to the PPP $N$. Consequently, $\Gamma$ is a.s. countable. Moreover, parts (i) and (ii) of Theorem 1.1 allow us to associate to each element $\left(\gamma_{1}, \gamma_{2}\right)$ of $\Gamma$ its common asymptotic direction. This provides a surjective function from $\Gamma$ onto the set $D$. Hence, $D$ is a.s. countable.

\section{Distribution of the $\theta(i, j)$ and conjectures}

In this section we provide some clues and conjectures that may help the understanding of the distribution of the vector $(\theta(1,2), \ldots, \theta(m-1, m), \theta(m, 1))$ of asymptotic directions of 
the interfaces, given that there are $m$ unbounded trees and assuming that the latter are labeled in the trigonometric sense. For this purpose, it is equivalent to study the distribution of the sectors $(\phi(i+1):=\theta(i+1, i+2)-\theta(i, i+1), i \in\{1, \ldots, m\})$ (with the convention that $\theta(m, m+1)=\theta(m, 1)$ and $\theta(m+1, m+2)=\theta(1,2))$, which characterize the asymptotic width of the unbounded trees.

Proposition 5.1. Conditionally on having $m$ unbounded trees, the angles between two interfaces are identically distributed with expectation $2 \pi / \mathrm{m}$.

Note first that this rules out the possibility that the asymptotic directions $\theta(i, j)$ are independent, uniform random variables on $[0,2 \pi)$. Otherwise, the distributions of the sectors would be beta distributions, $\mathbb{B}(1, m)$, whose expectation is $2 \pi /(m+1)$. There is thus interaction between the $\theta(i, j)$.

Our conjecture is as follows.

Conjecture 5.1. Conditionally on $m \in\{2,3,4,5\}$, the vector $(\phi(1), \ldots, \phi(m))$ has a distribution close to a symmetric Dirichlet distribution of order $m$ on $[0,2 \pi)$ with parameter $\alpha \neq 1, \operatorname{Dir}(m,[0,2 \pi), \alpha)$.

Symmetric Dirichlet distributions of order $m$ and parameter $\alpha>0$ on $[0,2 \pi)$ are probability distributions on $\mathbb{R}^{m}$ with support in $\Lambda=\left\{\eta=\left(\eta_{1}, \ldots, \eta_{m}\right) \in \mathbb{R}^{m}, \sum_{i=1}^{m} \eta_{i}=2 \pi\right\}$, and with the following density with respect to the Lebesgue measure on $\Lambda$ :

$$
f\left(\eta_{1}, \ldots, \eta_{m} ; \alpha\right)=\frac{1}{\mathbb{B}(\alpha)} \prod_{i=1}^{m}\left(\frac{\eta_{i}}{2 \pi}\right)^{\alpha-1} .
$$

Here

$$
\mathbb{B}(\alpha)=\frac{\left(\int_{0}^{+\infty} t^{\alpha-1} \mathrm{e}^{-t} \mathrm{~d} t\right)^{m}}{\int_{0}^{+\infty} t^{m \alpha-1} \mathrm{e}^{-t} \mathrm{~d} t}
$$

is the beta function. If we had a Dirichlet distribution conditionally on $m$, the marginal distribution of the exchangeable sectors would be a beta distribution $\mathbb{B}(\alpha,(m-1) \alpha)$ on $[0,2 \pi)$ with expectation $2 \pi / m$. This would also show that the distributions of the asymptotic directions $\theta(i, j)$ depend only on the number $m$ of unbounded trees and not on the number of offspring of $O$, which is a local phenomenon that is forgotten at large radii.

Let us illustrate the Conjecture 5.1 using simulations. We compute the angle between two interfaces and calibrate beta distributions. Whereas there are no closed-form formulae for the maximum-likelihood estimates, the moment estimates are as follows:

$$
\widehat{\alpha}=\frac{\bar{x}}{2 \pi}\left(\frac{\bar{x}(2 \pi-\bar{x})}{\operatorname{var}(x)}-1\right), \quad \widehat{\beta}=\frac{2 \pi-\bar{x}}{2 \pi}\left(\frac{\bar{x}(2 \pi-\bar{x})}{\operatorname{var}(x)}-1\right) .
$$

The different densities and the associated beta approximations are given in Figure 7.

We discuss the cases $m=2$ and $m=3$ for which a sufficiently large number of simulations are done to perform statistical tests.

Case 1: $m=2$. In this case, the joint law of $(\theta(1,2), \theta(2,1))$ is completely described by the distribution of one of the two sectors, say $\phi(1)$. Conditionally on the first interface $\theta(1,2)$, we can wonder whether the other interface is uniformly and independently distributed, i.e. whether $\phi(1)$ is a uniform random variable on $[0,2 \pi]$. Testing $H_{0}: \alpha=\beta=1$, and a likelihood ratio test provides a test statistic of 2274.93 , which leads us to reject the null assumption and, 


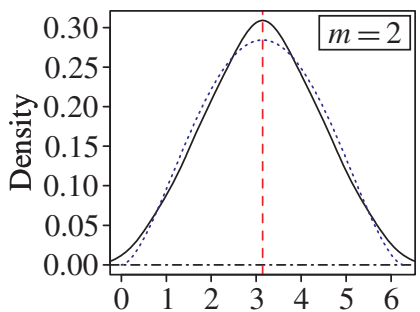

(a) $N=5868$, bandwidth $=0.3$

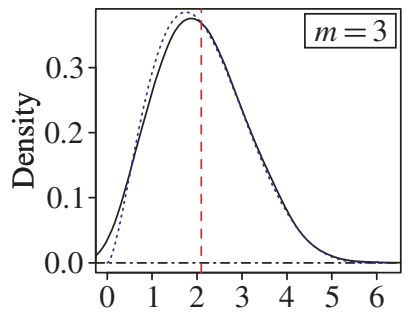

(b) $N=5196$, bandwidth $=0.3$

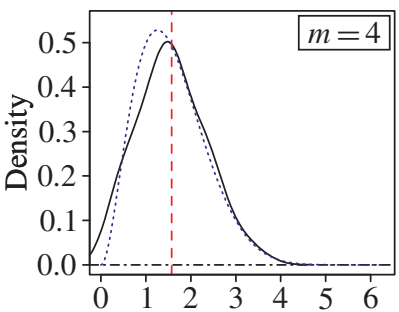

(c) $N=276$, bandwidth $=0.3$

FIGURE 7: Density estimation for the angles between two interfaces, given the number $m$ of unbounded trees. Given $m$, the distribution concentrates around $2 \pi / m$ (dashed lines) and has a smaller variance for larger $m$ values. Calibration with beta distributions have been carried out (dotted lines), but the KS test (with test statistic $D$ ) rejects the null hypothesis of the beta distribution $B(\widehat{\alpha},(m-1) \widehat{\beta})$ in the three cases with $p$-values smaller than $2.2 \mathrm{e}-16$, although the distributions look similar graphically. (a) $\widehat{\alpha}=\widehat{\beta}=2.74, D=96 \%$. (b) $\widehat{\alpha}=2.69, \widehat{\beta}=5.38, D=91.77 \%$. (c) $\widehat{\alpha}=2.99, \widehat{\beta}=8.99$, $D=88.79 \%$.

hence, the independence between the asymptotic direction of the two interfaces. We can easily be convinced of this by looking at Figure 7(a). As a consequence, the asymptotic directions $\theta(1,2)$ and $\theta(2,1)$ are not independent.

Case 2: $m=3$. In this case we performed a $\chi^{2}$-test to test the adequation of the joint distribution of the sectors to a Dirichlet distribution. Since the sum of the sectors is equal to $2 \pi$, we consider the couple $(\phi(1), \phi(2))$. With our simulations, the $\chi^{2}$-test statistic is equal to 176.49 and the adequation with the Dirichlet distribution is rejected. However, we can see that, as conjectured, the simulated sample looks like a simulated sample from a Dirichlet distribution.

\section{Appendix A. Noncrossing property for the paths of the RST}

Lemma A.1. Any two paths $\gamma$ and $\gamma^{\prime}$ of the RST (finite or not) cannot cross

$$
(X, \mathcal{A}(X)) \cap\left(X^{\prime}, \mathcal{A}\left(X^{\prime}\right)\right)=\varnothing \quad \text { for all } X \in \gamma \text { and all } X^{\prime} \in \gamma^{\prime}
$$

(where $(a, b)$ denotes the segment $[a, b]$ in $\mathbb{R}^{2}$ without its endpoints).

Proof. Let us assume that there exists a point $I$ belonging to both $(X, \mathcal{A}(X))$ and $(Y, \mathcal{A}(Y))$. It is easy to check that this assumption and the construction rule of the RST force $X, Y, \mathcal{A}(X)$, and $\mathcal{A}(Y)$ to be four different points. The same is true for their Euclidean norms with probability 1. Moreover, without loss of generality, we can also assume that $|Y|<|X|$. Then two cases can be distinguished.

Case 1. If $|\mathcal{A}(X)|<|Y|$ then $Y$ is closer to $\mathcal{A}(Y)$ than $\mathcal{A}(X):|\mathcal{A}(Y)-Y|<|\mathcal{A}(X)-Y|$. In the same way, the inequality $|\mathcal{A}(Y)|<|Y|<|X|$ implies that $|\mathcal{A}(X)-X|<|\mathcal{A}(Y)-X|$. Now, the triangular inequality leads to a contradiction:

$$
\begin{aligned}
|\mathcal{A}(Y)-Y|+|\mathcal{A}(X)-X| & <|\mathcal{A}(X)-Y|+|\mathcal{A}(Y)-X| \\
& <|\mathcal{A}(X)-I|+|I-Y|+|\mathcal{A}(Y)-I|+|I-X| \\
& <|\mathcal{A}(Y)-Y|+|\mathcal{A}(X)-X| .
\end{aligned}
$$

Case 2. We now assume that $|Y|<|\mathcal{A}(X)|$; see Figure 8. The points $X$ and $\mathcal{A}(X)$ do not belong to the open ball $B(O,|Y|)$ which contains $\mathcal{A}(Y)$ by definition. Hence, the existence 


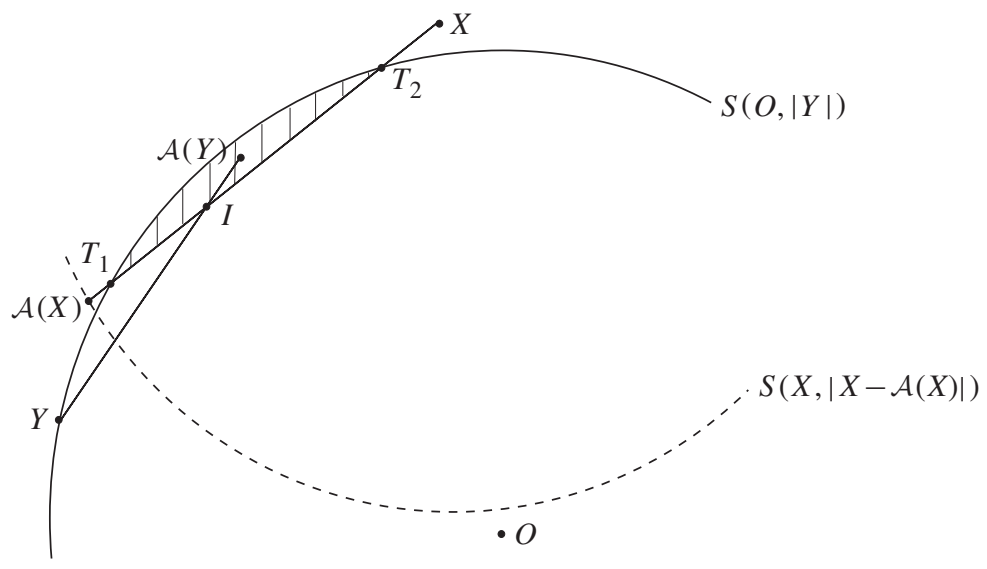

Figure 8: The hatched area corresponds to the one of the sets $U$ and $V$ which is included in the ball $B(X,|X-\mathcal{A}(X)|)$. Here, it contains $\mathcal{A}(Y)$. Note that the origin $O$ cannot belong to the ball

$$
B(X,|X-\mathcal{A}(X)|) \text {. }
$$

of the point $I$ forces the segment $(X, \mathcal{A}(X))$ to intersect $S(O,|Y|)$ at two distinct points, say $T_{1}$ and $T_{2}$, dividing the closed ball $\bar{B}(O,|Y|)$ into two nonoverlapping sets, say $U$ and $V$. By hypothesis, each of these two sets contains (exactly) one of the points $Y$ and $\mathcal{A}(Y)$. Since $\left|T_{1}-X\right|$ and $\left|T_{2}-X\right|$ are smaller than $|X-\mathcal{A}(X)|$ by construction, one of the regions $U$ or $V$ is included in the ball $B(X,|X-\mathcal{A}(X)|)$. So, one of the points $Y$ and $\mathcal{A}(Y)$ belongs to the ball $B(X,|X-\mathcal{A}(X)|)$. This contradicts the fact that $\mathcal{A}(X)$ is the ancestor of $X$.

\section{Acknowledgements}

This work was financed by the GdR 3477 Géométrie Stochastique. The authors thank the members of the 'Groupe de travail Géométrie Stochastique' at the Université Lille 1 and J.-B. Gouéré for enriching discussions.

\section{References}

[1] Athreya, S., Roy, R. and Sarkar, A. (2008). Random directed trees and forest-drainage networks with dependence. Electron. J. Prob. 13, 2160-2189.

[2] Baccelli, F. and Bordenave, C. (2007). The radial spanning tree of a Poisson point process. Ann. Appl. Prob. 17, 305-359.

[3] Bonichon, N AND Marckert, J.-F. (2011). Asymptotics of geometrical navigation on a random set of points in the plane. Adv. Appl. Prob. 43, 889-942.

[4] Coupier, D. (2011). Multiple geodesics with the same direction. Electron. Commun. Prob. 16, 517-527.

[5] Coupier, D. And Heinrich, P. (2011). Stochastic domination for the last passage percolation tree. Markov Process. Relat. Fields 17, 37-48.

[6] Coupier, D. And Heinrich, P. (2012). Coexistence probability in the last passage percolation model is 6-8 $\log 2$. Ann. Inst. H. Poincaré Prob. Statist. 48, 973-988.

[7] CoupIER, D. AND Tran, V. C. (2013). The 2D-directed spanning forest is almost surely a tree. Random Structures Algorithms 42, 59-72.

[8] Ferrari, P. A. and Pimentel, L. P. R. (2005). Competition interfaces and second class particles. Ann. Prob. 33, 1235-1254.

[9] Gangopadhyay, S., Roy, R. And Sarkar, A. (2004). Random oriented trees: a model of drainage networks. Ann. App. Prob. 14, 1242-1266.

[10] Howard, C. D. And Newman, C. M. (1997). Euclidean models of first-passage percolation. Prob. Theory Relat. Fields 108, 153-170. 
[11] Howard, C. D. and Newman, C. M. (2001). Geodesics and spanning trees for Euclidean first-passage percolation. Ann. Prob. 29, 577-623.

[12] JACOD, J. And Shiryaev, A. N. (1987). Limit Theorems for Stochastic Processes. Springer, Berlin.

[13] Norris, J. ANd Turner, A. G. (2012). Hastings-Levitov aggregation in the small-particle limit. Commun. Math. Phys. 316, 809-841.

[14] Pimentel, L. P. R. (2007). Multitype shape theorems for first passage percolation models. Adv. Appl. Prob. 39, 53-76.

[15] Talagrand, M. (1995). Concentration of measure and isoperimetric inequalities in product spaces. Inst. Hautes Études Sci. Publ. Math. 81, 73-205. 